\title{
Deep learning prediction of mild cognitive impairment conversion to Alzheimer's disease at 3 years after diagnosis using longitudinal and whole-brain 3D MRI
}

\author{
Ethan Ocasio ${ }^{1}$, Tim Q Duong ${ }^{\text {Corresp. } 1}$ \\ ${ }^{1}$ Department of Radiology, Montefiore Medical Center, Albert Einstein College of Medicine, Bronx, New York, United States \\ Corresponding Author: Tim Q Duong \\ Email address: tim.duong@einsteinmed.org
}

Background. While there is no cure for Alzheimer's disease (AD), early diagnosis and accurate prognosis of $A D$ may enable or encourage lifestyle changes, neurocognitive enrichment, and interventions to slow the rate of cognitive decline. The goal of our study was to develop and evaluate a novel deep learning algorithm to predict mild cognitive impairment $(\mathrm{MCl})$ to $A D$ conversion at three years after diagnosis using longitudinal and whole-brain 3D MRI.

Methods. This retrospective study consisted of 320 normal cognition (NC), $554 \mathrm{MCl}$, and $237 \mathrm{AD}$ patients. Longitudinal data include T1-weighted 3D MRI obtained at initial presentation with diagnosis of $\mathrm{MCl}$ and at 12-month follow up. Whole-brain 3D MRI volumes were used without a priori segmentation of regional structural volumes or cortical thicknesses. MRIs of the AD and NC cohort were used to train a deep learning classification model to obtain weights to be applied via transfer learning for prediction of $\mathrm{MCl}$ patient conversion to AD at three years post-diagnosis. Two (zero-shot and fine tuning) transfer learning methods were evaluated. Three different convolutional neural network (CNN) architectures (sequential, residual bottleneck, and wide residual) were compared. Data were split into 75\% and 25\% for training and testing, respectively, with 4-fold cross validation. Prediction accuracy was evaluated using balanced accuracy. Heatmaps were generated.

Results. The sequential convolutional approach yielded slightly better performance than the residualbased architecture, the zero-shot transfer learning approach yielded better performance than fine tuning, and CNN using longitudinal data performed better than CNN using a single timepoint MRI in predicting $\mathrm{MCl}$ conversion to $\mathrm{AD}$. The best CNN model for predicting $\mathrm{MCl}$ conversion to $\mathrm{AD}$ at three years after diagnosis yielded a balanced accuracy of 0.793 . Heatmaps of the prediction model showed regions most relevant to the network including the lateral ventricles, periventricular white matter and cortical gray matter.

Conclusions. This is the first convolutional neural network model using longitudinal and whole-brain 3D MRIs without extracting regional brain volumes or cortical thicknesses to predict future $\mathrm{MCI}$ to $\mathrm{AD}$ conversion at 3 years after diagnosis. This approach could lead to early prediction of patients who are likely to progress to AD and thus may lead to better management of the diseases. 


\section{Deep Learning Prediction of Mild Cognitive}

2 Impairment Conversion to Alzheimer's Disease at 3

3 years after diagnosis using Longitudinal and Whole4 Brain 3D MRI

5

6

7

8

9

10

11

12

13

14

18
Ethan Ocasio ${ }^{1}$, Tim Q Duong ${ }^{1}$

${ }^{1}$ Department of Radiology, Montefiore Medical Center, Albert Einstein College of Medicine, Bronx, New York

Corresponding Author:

Tim Duong, $\mathrm{PhD}^{1}$

Department of Radiology, Albert Einstein College of Medicine, 111 E 210th St, Bronx, New York 10467, Email address: Tim.duong@einsteinmed.org 
19

20

21

22

23

24

25

26

27

28

29

30

31

32

33

34

35

36

37

38

39

40

41

42

43

44

45

46

47

48

49

50

51

52

53

54

\section{Abstract}

Background. While there is no cure for Alzheimer's disease (AD), early diagnosis and accurate prognosis of AD may enable or encourage lifestyle changes, neurocognitive enrichment, and interventions to slow the rate of cognitive decline. The goal of our study was to develop and evaluate a novel deep learning algorithm to predict mild cognitive impairment (MCI) to AD conversion at three years after diagnosis using longitudinal and whole-brain 3D MRI.

Methods. This retrospective study consisted of 320 normal cognition (NC), 554 MCI, and 237 AD patients. Longitudinal data include T1-weighted 3D MRI obtained at initial presentation with diagnosis of MCI and at 12-month follow up. Whole-brain 3D MRI volumes were used without a priori segmentation of regional structural volumes or cortical thicknesses. MRIs of the AD and $\mathrm{NC}$ cohort were used to train a deep learning classification model to obtain weights to be applied via transfer learning for prediction of MCI patient conversion to AD at three years postdiagnosis. Two (zero-shot and fine tuning) transfer learning methods were evaluated. Three different convolutional neural network $(\mathrm{CNN})$ architectures (sequential, residual bottleneck, and wide residual) were compared. Data were split into $75 \%$ and $25 \%$ for training and testing, respectively, with 4-fold cross validation. Prediction accuracy was evaluated using balanced accuracy. Heatmaps were generated.

Results. The sequential convolutional approach yielded slightly better performance than the residual-based architecture, the zero-shot transfer learning approach yielded better performance than fine tuning, and CNN using longitudinal data performed better than CNN using a single timepoint MRI in predicting MCI conversion to AD. The best CNN model for predicting MCI conversion to $\mathrm{AD}$ at three years after diagnosis yielded a balanced accuracy of 0.793 . Heatmaps of the prediction model showed regions most relevant to the network including the lateral ventricles, periventricular white matter and cortical gray matter.

Conclusions. This is the first convolutional neural network model using longitudinal and wholebrain 3D MRIs without extracting regional brain volumes or cortical thicknesses to predict future MCI to AD conversion at 3 years after diagnosis. This approach could lead to early prediction of patients who are likely to progress to AD and thus may lead to better management of the diseases. 
55

56

57

58

59

60

61

62

63

64

65

66

67

68

69

70

71

72

73

74

75

76

77

78

79

80

81

82

83

84

85

86

87

88

89

90

91

92

93

94

95

\section{Background}

Alzheimer's disease (AD) is a progressive neurodegenerative disease characterized by loss of memory and other cognitive functions (McKhann et al. 2011). Mild Cognitive Impairment (MCI) is considered a transitional state between normal aging and dementia. Many patients progress from MCI to AD, but others remain stable without developing AD. Although diagnoses of MCI and AD are typically made using neuropsychological tests (Petersen et al. 1999; Jak et al. 2009), imaging methods are also used to diagnose $\mathrm{AD}$ and to monitor disease progression because they provide neural correlates of the underlying brain dysfunction in a longitudinal non-invasive manner (Johnson et al. 2012). While there is no cure for AD, early diagnosis and accurate prognosis may enable or encourage lifestyle changes, neurocognitive enrichment, and therapeutic interventions that strive to improve symptoms, or at least slow down mental deterioration, thereby improving the quality of life (Epperly et al. 2017).

Machine learning (ML) is increasingly being used in medicine from disease classification to prediction of clinical progression (de Bruijne 2016; Erickson et al. 2017). ML uses algorithms to learn the relationship amongst different data elements to inform outcomes. Neural networks, a form of ML, are made up of a collection of connected nodes that model the neurons present in a human brain (Graupe 2013). Each connection, similar to a synapse, transmits and receives signals to other nodes. Each node and the connections it forms are initialized with weights which are adjusted throughout training and create mathematical relationships between the input data and the outcomes. In contrast to traditional analysis methods such as logistic regression, neural networks do not require relationships between different input variables and the outcomes to be explicitly specified a priori. In radiology, ML can accurately detect lung nodules on chest X-rays (Harris et al. 2019). In cardiology, ML can detect abnormal EKG patterns (Johnson et al. 2018). ML has also been used to estimate risk, such as in the Framingham Risk Score for coronary heart disease (Alaa et al. 2019), and to guide antithrombotic therapy in atrial fibrillation (Lip et al. 2010) and defibrillator implantation in hypertrophic cardiomyopathy (O'Mahony et al. 2014). Convolutional neural networks (CNNs), a deep-learning method, are widely used for image analysis and analysis of complex data (Lecun et al. 1998; Krizhevsky et al. 2012; Simonyan \& Zisserman 2014).

Deep learning classification amongst normal cognition (NC), MCI and AD based on magnetic resonance imaging (MRI) data have been reported (Cheng et al. 2017; Korolev et al. 2017; Wen et al. 2020). By contrast, there are comparatively fewer studies that reported prediction of MCI to AD conversion using deep learning of MRI data (Lian et al. 2018; Lin et al. 2018; Liu et al. 2018; Shmulev \& Belyaev 2018; Basaia et al. 2019; Wen et al. 2020). A few ML studies used extracted brain structures or cortical thicknesses, and some used 3D patches from predetermined locations across the brain, but not whole-brain MRI data, to predict MCI to AD conversion (Lian et al. 2018; Liu et al. 2018; Wen et al. 2020). Most of the few prediction studies used single timepoint MRI data. To our knowledge there are only two studies that predicted disease 
96 progression using longitudinal imaging data. Bhagwat used a neural network (albeit not deep 97 learning) and extracted cortical thicknesses from MRIs at two time points to predict decline in 98 Mini-Mental Status Exam (MMSE) scores (Bhagwat et al. 2018). Ostertag et al. used a CNN 99 model on whole-brain MRI at two time points to predict decline in MMSE score but did not test 100 their model on an independent testing dataset (Ostertag et al. 2019). These two studies mixed $101 \mathrm{NC}, \mathrm{MCI}$ and $\mathrm{AD}$ participants and thus accuracies are not applicable to prediction of MCI to AD 102 conversion. To our knowledge, there are no published studies to date on deep learning to predict

103 104

\section{$M C I$ to AD conversion using longitudinal and whole-brain $3 D$ MRI.}

The goal of our study was thus to develop and evaluate a novel deep-learning algorithm to predict $\mathrm{MCI}$ to $\mathrm{AD}$ conversion at three years after diagnosis using longitudinal and whole-brain 3D MRI. Longitudinal data include MRI obtained at initial presentation with diagnosis of MCI and at 12-month follow up. Whole-brain 3D MRI volumes were used without a priori segmentation of regional structural volumes or cortical thicknesses. Several convolutional model architectures, transfer learning methods, and methods of merging longitudinal whole-brain 3D MRI data were evaluated to derive the final optimal deep-learning predictive model.

\section{Methods}

Figure 1 shows the overall design of the experiment. 3D MRIs of the AD and NC cohort were trained in a CNN classification model to obtain weights for transfer learning to be used in the $\mathrm{CNN}$ prediction of MCI patient conversion to $\mathrm{AD}$ in 3 years after diagnosis. Two (zero-shot and fine tuning) transfer learning methods for prediction were evaluated (Pan \& Yang 2010). The zero-shot transfer method used the intact weights obtained from the NC-AD classification without any additional training. The fine-tuning transfer method kept the weights in the convolutional layers frozen while allowing the remaining fully connected layers to change during additional training against the MCI images.

\section{Participants}

Data used in this study was obtained from the Alzheimer's Disease Neuroimaging Initiative (ADNI) database (adni.loni.usc.edu). Patients were taken from the ADNI1, ADNIGO, ADNI2, and ADNI3 patient sets. For the prediction task, inclusion criteria were patients diagnosed with MCI at baseline with MRI taken at baseline and $\sim 12$ months after baseline, and with a final diagnosis at 3 years post baseline of either MCI (labeled as stable MCI or sMCI) or AD (labeled as progressive MCI or pMCI). Patients who converted from MCI to AD before their 12-month follow up image were excluded since the analysis of their longitudinal image at that point would represent a diagnostic classification not a prediction. Table 1 summarizes the participant demographics. Data were split into $75 \%$ and $25 \%$ for training/validation and testing, respectively, with the training/validation set composed of 415 patients (249 sMCI and 166 pMCI), and the testing set composed of 139 patients ( $84 \mathrm{sMCI}$ and $55 \mathrm{pMCI})$. Then, we optimized the networks using a 4-fold cross-validation on the training/validation set, resulting in 
136

137

138

139

140

141

142

143

144

145

146

147

148

149

150

151

152

153

154

155

156

157

158

159

160

161

162

163

164

165

166

167

168

169

170

171

172

173

174

175

$56.25 \%$ for training and $18.75 \%$ for validation from the complete data in each fold split. In the end, we had four trained models for each experiment configuration, and reported the mean and standard deviation (SD) BA.

For the AD-NC classification to obtain weights for transfer learning, a separate group of participants with a baseline diagnosis of AD or NC with images taken at baseline and $\sim 12$ months from baseline were selected. Data for training/validation and testing were randomly split up as $70 \%$ and $30 \%$ respectively. The training/validation set consisted of 387 patients (160 AD and $227 \mathrm{NC}$ ), while the independent testing set consisted of 170 patients (77 AD and $93 \mathrm{NC}$ ). Using 4-fold cross validation on the training/validation set resulted in 52.5\% for training and $17.5 \%$ for validation from the complete data in each fold split. The networks were trained to classify the patients between $\mathrm{AD}$ and $\mathrm{NC}$ against the ground truth diagnosis of each patient using ADNI criteria. For all experiments the study size represents the total available patients in ADNI database fully meeting all inclusion criteria.

\section{Preprocessing}

3D volumes of T1-weighted MRI were used as input to the networks. To remove intensity inhomogeneity from the image inputs, T1-weighted images with nonparametric non-uniform intensity normalization (N3) correction (Sled et al. 1998) were selected from the ADNI database. All MRIs were skull stripped with DeepBrain (Itzcovich 2018), then linearly registered against a $2 \mathrm{~mm}$ standard brain with nine degrees of freedom (translation, rotation, scaling) using FSL FLIRT (Jenkinson et al. 2012), and finally $\mathrm{min} / \mathrm{max}$ intensity normalized. Resulting images had a resolution of 91x109x91 voxels. During training, data augmentation was performed on the training set by rotating each MRI by up to $5 \%$ in any direction and randomly flipping them left to right along the sagittal axis.

\section{Training, validation, and testing}

Images were split into testing, validation, and testing sets at the patient level in order to avoid data leakage. For both classification and prediction tasks, after assigning labels (either AD vs NC or sMCI vs pMCI), SciPy's train_test_split function was used to randomly split training/validation and testing and cross validation (Virtanen et al. 2020). Stratification was done based on the labels in order to maintain a consistent distribution of diagnoses across the training, validation, and testing datasets. Randomization seed was set up as a constant to ensure the same train/validation/test split was obtained for each experiment run. Balanced accuracy (BA), defined as the average of sensitivity and specificity, was used as the main binary classifier metric to eliminate the inflated accuracy effect caused by imbalanced data sets. For purposes of computing accuracy, we use a standard value of 0.5 as the threshold between the two labels (either NC \& $\mathrm{AD}$ or $\mathrm{pMCI} \& \mathrm{sMCI})$. We also computed the area under the receiver operating characteristic curve (AUC) for each run, since it provides a more general measure of the potential performance of a network across a range of thresholds. To prevent data leakage, once the test partition was 
176 randomly selected, the test set images were set aside and not used for any training or validation.

177 We repeated each training experiment 4 times, using the standard k-fold cross validation

178 approach where the training/validation set is partitioned into four subsets, using each subset once

179 for validation, and reported both the mean and standard deviation of the BAs for each

180 experiment. This ensures that each image is included at least once in both the validation and

181 training sets, minimizing the potential selection bias that a single random data split may

182 introduce. For each cross-validation fold, classification task training was performed for 200

183 epochs with early stopping based on no improvement in loss function for 80 epochs. Fine tuning

184 after freezing all convolutional layers was performed for 100 epochs with early stopping patience

185 of 40 . The weights of the epoch ending with the lowest loss were saved and used to obtain the

186 validation BA, and then the network was run against the test set for the test BA. Additional

187 attempts at fine tuning by also unfreezing the last convolutional block were noted to degrade

188 accuracy, so this approach was not considered any further.

189

190

191

192

\section{CNN Architecture}

The neural network models (Figure 2) consisted of a convolutional section followed by a fully connected section (head). Three (sequential, residual with bottleneck, and wide residual) types of convolutional blocks (Figure 3) and three head architectures (Figure 4) were examined. In

194 addition, we also evaluated these networks using a single (baseline) timepoint MRI and MRIs at two time points (baseline and 12 months). For the dual timepoint networks, three types of networks were explored to incorporate longitudinal data: (1) Siamese network (two identical parallel channels with weights tied together) using a subtraction layer as the merging function, (2) Siamese network with a concatenation merge layer, and (3) a twin network (identical channels with weights independently optimized). Since the flattened set of post-convolution features in the twin architecture is different in each channel, as they are the result of different parameters, there is no rationale for directly subtracting them, so we only considered a

203 concatenation merge option for the twin architecture. For all networks, the final binary classifier layer was fully connected with sigmoid activation. When performing the prediction tasks (using both zero-shot learning and fine-tuning) in the single timepoint experiments, we attempted the same task using both the initial baseline MRI as well as the 1-year follow up image. Using either the single timepoint with the 1-year image or both timepoints together longitudinally represents, for patients who have had $\mathrm{MCI}$ for one year and not yet progressed to $\mathrm{AD}$, a prediction of 209 whether they will eventually convert to $\mathrm{AD}$ within two more years.

After initial experimentation, an optimal set of blocks was identified for the sequential and wide residual network styles, namely using 6 blocks with widths (number of activation maps) $=\{64$,

214 $128,256,512,1024,2048\}$. In the case of the sequential convolution network, each block reduced the resolution via maximum pooling as the width increased, down to $1 \times 1 \times 1$ in the final convolutional block. In the case of the wide residual network, convolutions with the use of strides gradually reduced the resolution down to $2 \times 2 \times 2$, with a global maximum pooling layer in 
216 the head portion of the network. When a convolutional layer processes an input whose size is 217 odd-numbered in any of its dimensions ( $2 n-1$ for any integer $n$ ) the resulting output of a stride 2 218 convolution with zero-padding will be of size $n$ for the corresponding dimension. For example, 219 since the input image has resolution $91 \times 109 \times 91$, the output after the first stride 2 convolution

220

221

222

223

224

225

226

227

228

229

230

231

232

233

234

235

236

237

238

239

240

241

242

243

244

245

246

247

248

249

250

251

252

253

254 will be $46 \times 55 \times 46$. For the bottleneck residual, the final convolutional resolution was also $2 \times 2 \times 2$, achieved via strides, but this required 7 blocks with widths $=\{64,64,128,256,512,1024$, $2048\}$. The bottleneck architecture used $1 \times 1 \times 1$ convolutions for resolution reduction, so the behavior was slightly different when the resolution had odd numbers, allowing for 7 instead of 6 blocks until the resolution was down to $2 \times 2 \times 2$. The portion of the network with flattened nonconvolutional fully connected layers after the last convolutional layer up until the final binary classifier is known as the "head." After initial analysis of networks with varying heads, a global maximum pooling operation resulting in a fully connected layer with a number of nodes equal to the number of activation maps (width) of the last convolutional step, followed directly by a single final dense prediction layer, was selected as the optimal fully connected layer architecture (Figure 4A).

Training was initially attempted using both non-adaptive (SGD with Nesterov momentum), as well as adaptive (Adam) optimizers (Kingma \& Ba 2019). The Adam optimizer was able to achieve reductions in loss function with accompanying increase in accuracy much more rapidly and aggressively. However, without a scheduled reduction of the base learning rate, the network became unstable in the latter epochs with rapid swings in the loss function. The use of an exponentially decaying learning rate schedule consistently stabilized both the loss and accuracy curves in an optimal fashion. The final selected optimization approach was thus the Adam optimizer with an exponentially decreasing learning rate schedule with expected initial rate $L$ $R_{\text {start }}$ and final rate $L R_{\text {end }}$ where $t$ is the current epoch and $T$ is the final expected number of epochs:

(1)

$$
L R_{\text {epoch }}=L R_{\text {start }}\left(\frac{L R_{\text {end }}}{L R_{\text {start }}}\right)^{t / T}
$$

L2 regularization as also added in the convolutional layers in all networks. For the sequential model, we used a regularization parameter of 0.005 , and for the residual models we used a parameter of 0.0001 . All training was performed using Tensorflow2/Keras python library, on Google Compute Platform virtual instances with Tesla V-100 GPU acceleration.

\section{Network visualization by heatmap}

To visualize the brain regions that are most relevant to the network, the Grad-CAM (Selvaraju et al. 2017) technique was modified to work in 3 dimensions for generating heatmaps. Since the models reduced the resolution of the image information within the convolution blocks down to $2 \times 2 \times 2$ voxels or less, the 3D Grad-CAM technique was applied to higher convolutional layers 
255

256

257

258

259

260

261

262

263

264

265

266

267

268

269

270

271

272

273

274

275

276

277

278

279

280

281

282

283

284

285

286

287

288

289

290

291

292

(with resolution close to 40 voxels per axis) to obtain more useful visualization heatmaps. This approach enabled visual highlighting of the sections of the images that were most significant to the network. Heatmaps were obtained during the execution of the prediction models.

\section{Results}

AD versus $\mathrm{NC}$ classification to generate weights

Figure 5 shows training curves for sequential single channel and wide residual dual channel training for the classification experiments. Overall, loss functions converged and leveled off at around 75 epochs. Other models showed similar convergence characteristics. Table 2 details the results of the classification experiments to generate weights. For the single timepoint networks, sequential architecture performed best $(\mathrm{BA}=0.860)$ followed by wide residual $(\mathrm{BA}=0.840)$ and bottleneck residual $(B A=0.727)$ on the testing data. For the dual timepoint networks, Siamese network with subtraction performed poorly overall with all architectures (BA $0<0.65$ ) and that the twin non-Siamese approach with merge concatenation performed best for dual channels. The wide residual $(\mathrm{BA}=0.887)$ performed best followed by sequential $(\mathrm{BA}=0.876)$ and bottleneck residual $(\mathrm{BA}=0.800)$. Training time for each run was approximately $60-90$ minutes. After model was trained, classification of a patient takes two seconds or less (most of this time is loading the images from storage into memory).

Prediction of AD conversion at 3 years after diagnosis

Figure 6 shows the training curve for one of the dual sequential transfer learning fine-tuning attempts for the prediction experiment. Additional training did not improve the accuracy even though there was some clear reduction in loss function during training. Other models showed similar characteristics. Table 3 summarizes the results of the prediction experiments, showing the outcomes of the dual and single timepoint networks using both zero-shot and fine-tuning transfer learning. The bottleneck residual convolutional style, because it performed much worse for classification, was not considered for prediction. For the single timepoint experiments, the 12-month images performed better than the initial baseline images during the classification task. The dual timepoint networks performed better than the single timepoints. For zero-shot best results were obtained from the sequential model, with the dual sequential producing a 0.795 average BA against the test set, followed by the single timepoint sequential (using the 1-year image as input) with a BA of 0.774 . Transfer learning with fine tuning in all cases resulted in lowered accuracy as compared with the zero-shot approach. This occurred whether fine tuning was attempted by unfreezing only the fully connected layers or also the last convolutional layer. Training time for each fine-tuning experiment was approximately 15-30 minutes. Prediction of conversion took using a trained model (either zero-shot or fine-tuned) took two seconds or less. 
293 To visualize the brain regions that are most relevant to ML algorithms, post-training heatmaps

294 for the wide residual dual network were generated (Figure 7). The highlighted structures

295 included the lateral ventricles, periventricular white matter, and cortical surface gray matter.

296

297

298

299

300

301

302

303

304

305

306

307

308

309

310

311

312

313

314

315

316

317

318

319

320

321

322

323

324

325

326

327

328

329

330

331

332

\section{Discussion}

This study developed and evaluated a few sophisticated ML algorithms to predict which MCI patients would convert to $\mathrm{AD}$ at three years post-diagnosis using longitudinal whole-brain 3D MRI without a priori segmentation of regional structural volumes or cortical thicknesses. MRI data used for prediction were obtained at baseline and one year after baseline. The sequential convolutional approach yielded slightly better performance than the residual-based architecture, the zero-shot transfer learning approach yielded better performance than fine tuning, and the $\mathrm{CNN}$ using longitudinal data performed better than the CNN using a single timepoint MRI in predicting $\mathrm{MCI}$ conversion to $\mathrm{AD}$. The best $\mathrm{CNN}$ model for predicting $\mathrm{MCI}$ conversion to $\mathrm{AD}$ at 3 years after diagnosis yielded a BA of 0.793 .

Our predictive model used whole-brain MRIs without extract regional brain volumes and cortical thicknesses. We also evaluated multiple longitudinal network configurations (i.e., Siamese and non-Siamese twin networks with subtraction and concatenation as the merge function).

Longitudinal images were found to be optimally processed by a twin architecture with concatenation merge. The dual timepoint network performed better regardless of whether the initial or the follow up image was used for the single timepoint. Restricting the network in a Siamese configuration where the weights of both channels are identical or using a subtraction merge function resulted in worse prediction, which suggests that the networks take full advantage of the additional information provided by the second time point data when they were allowed to train each channel with separate weights.

We employed 3D MRI instead of 2D multi-slice MRI. Previous studies have also reported MCI to $\mathrm{AD}$ prediction using a sequential full volume $3 \mathrm{D}$ architecture have obtained $\mathrm{BAs}$ of 0.75 (Basaia et al. 2019) and 0.73 (Wen et al. 2020), while a study using residual architecture showed a resulting BA of 0.67 (Shmulev \& Belyaev 2018) but did not involve longitudinal MRI data. Some studies used predetermined 3D patches uniformly sampled across the brain (Lian et al. 2018; Liu et al. 2018; Wen et al. 2020). A limitation of the 3D-patch approach is that a subsequent fusion of the results via some kind of ensemble or voting method is needed to obtain a subject-level prediction, and brain-wide anatomic relationships are not taken into account. There are two previous related studies that used longitudinal MRI data for prediction of MCI or AD disease progression. Bhagwat et al. employed baseline and 1-year MRIs with Siamese neural network with concatenation merge to predict a pattern of decline in patients' MMSE score, yielding an accuracy of 0.95 (Bhagwat et al. 2018). In contrast to our study, regional cortical thicknesses, a non-convolutional method, and clinical variables were used. The use of clinical 
333 variables could have contributed substantially to higher accuracy. Ostertag et al. used a similar 334 Siamese network but employed whole-brain MRI to predict decline in patients' MMSE score, 335 yielding a validation accuracy of 0.90 , but no independent evaluation on a separate test dataset 336 was performed (Ostertag et al. 2019). Moreover, these two studies differed from ours in that they 337 mixed $\mathrm{AD}, \mathrm{NC}$, and $\mathrm{MCI}$ patients together, and thus their prediction accuracies are not directly 338 comparable to those from MCI to AD conversion studies (thus accuracies are not applicable to 339 prediction of $\mathrm{MCI}$ to $\mathrm{AD}$ conversion) because the baseline diagnosis of $\mathrm{NC}$ or $\mathrm{AD}$ by itself is a

340

341

342

343

344

345

346

347

348

349

350

351

352

353

354

355

356

357

358

359

360

361

362

363

364

365

366

367

368

369

370

371

372 strong predictor of neurocognitive decline.

The use of a Siamese network architecture to analyze longitudinal changes in disease progression from medical images was explored by Li et al. and specifically studied in AD brain MRIs by Bhagwat et al. and Ostertag et al. (Bhagwat et al. 2018; Ostertag et al. 2019; Li et al. 2020). The idea behind Siamese networks is that both images are processed by the convolutional layers with identical parameters, with equivalent flattened sets of features for each image at the end of the convolutions. Thus, theoretically, a direct subtraction merge of the corresponding flattened features would represent a measure of the progression of the images - presumably an MCI patient whose structural MRI features have worsened in a year would be more likely to progress to $\mathrm{AD}$ than a patient whose features remain stable. However, a simple subtraction merge may result in loss of predictive information if there are particular features that are predictive of progression regardless of whether they have changed between baseline and 1-year. Thus, we also explore the concatenation merge. In addition, a twin (non-Siamese) network with separate parameters may provide, partly due to the additional power of doubling the number of convolutional parameters, better predictive capacity, so we also explored this architecture. Since the flattened set of post-convolution features in the twin architecture is different in each channel, as they are the result of different parameters, there is no rationale for directly subtracting them, so we only considered a concatenation merge option for the twin architecture.

Although for the initial classification task, the twin wide residual network performed best among all architectures, after the transfer learning the twin sequential network was the overall best performer. In the single channel variants, the sequential networks performed best. The bottleneck variant of the residual network performed the worst amongst all architectures. In general, the residual networks provide the benefit of reducing the vanishing gradient problem, as compared with a non-residual sequential style. The bottleneck in particular is meant to strongly prevent vanishing gradients. Since vanishing gradients did not appear significantly during training, the advantages of the residual network appeared not to materialize, and thus, overall, the sequential networks seemed best fit for 3D MRI whole-brain analysis.

\section{Heatmaps}

Heatmaps enabled visualization of the brain regions that were most relevant to ML algorithms to predict $\mathrm{MCI}$ and $\mathrm{AD}$ conversion. The most salient structures on the heatmaps were the lateral 
373 ventricles, periventricular deep white matter as well as extensive cortical gray matter. Ventricular

374 enlargement and atrophy are known to be associated with AD. Reduction in white-matter volume

375 has been described in $\mathrm{AD}$, including some the specific regions that our heatmap analysis found to

376 be on interest (Smith et al. 2000; Guo et al. 2010; Kao et al. 2019), including the cingulate gyrus

377 (Brun \& Gustafson 1976; Hirono et al. 1998; Jones et al. 2006), the middle occipital gyrus

378 (Zhang \& Wang 2015), and the putamen (Pini et al. 2016). Other brain regions that have shown

379 to be associated with development of $\mathrm{AD}$, such as the default node network and hippocampus,

380 are not uniformly highlighted in the heatmaps. Our analysis approach is different from previous

381

382

383

384

385

386

387

388

389

390

391

392

393

394

395

396

397

398

399

400

401

402

403

404

405

406

407

408

409

410

411 analysis and does not specifically identify networks, although amongst the heatmaps shown, there were components that were part of the default mode networks and hippocampus. In other words, our analysis did not specifically test whether hippocampus or default mode networks are predictive of MCI to AD conversion. It is possible that, given our MRI is based on structural changes, hippocampus and default mode networks might not have developed atrophy to be informative to prediction conversion.

\section{Other technical considerations}

We examined three different convolutional architectures to identify the best performance prediction model. Two residual variants were compared, with the wide residual network performing better than the bottleneck variant, and the non-residual sequential network performing better than both residual types. The two residual approaches compared here were 3D modifications of ResNet (He et al. 2016). The bottleneck variation used pre-activation, a technique where the batch normalization and activation layers precede the convolutions. The term "bottleneck" refers to a design where each residual block includes two initial layers with narrower widths. The second residual variant examined for comparison was the wide residual network (Zagoruyko \& Komodakis 2016). In this approach the widths were progressively increased, with an additional dropout layer between two convolutions in each residual block. The sequential model we tested was a 3D extension of the 2D VGG model (Simonyan \& Zisserman 2014), with sequential blocks formed by a combination of convolutional layers followed by pooling layers.

We also examined the relative performance of two transfer learning approaches. Zero-shot technique performed better than fine tuning. Further fine-tuning with the sMCI vs pMCI data reduced the accuracy of the prediction network from that obtained via the exclusive use of AD vs $\mathrm{NC}$ data for classification task training. The lack of training power of the MCI data suggests that brain images with either $\mathrm{AD}$ or $\mathrm{NC}$, with their more discriminant anatomic features, are more suited for training a network eventually used for detecting the more subtle distinctions between pMCI and sMCI.

Peer] Comput. Sci. reviewing PDF | (CS-2020:10:54832:2:0:NEW 26 Apr 2021) 
412 We also carefully prevented data leakage by splitting the training and testing datasets at patient 413 level, ensuring that no data from the same patient would end up in both groups (Wen et al. 2020). 414 Another type of leakage we avoided occurs when data are used for training the classification are 415 also used for prediction task. Finally, in this study the testing set results were collected only after 416 all training was completed to prevent a third possible kind of leakage, namely where results from 417 the test set influence the selection of hyperparameters or architecture. We also excluded patients 418 who converted from MCI to AD before their 12-month follow up.

419

420

421

422

423

424

425

426

427

428

429

430

431

432

433

434

435

436

437

438

439

440

441

442

443

444

445

446

447

448

449

450

451

In several cases for both classification and prediction we observed that the BA for the testing dataset had a slightly higher mean and lower standard deviation than the corresponding results for validation. This higher variation in the validation experiments could potentially be explained by the fact that each cross-validation fold has a different validation set of images while all the testing results are obtained from the same single test set applying the different trained models. This higher variation also means that a single lower BA result in one of the validation folds could pull down the mean validation BA.

\section{Limitations and future directions}

The increase in BA obtained by using the longitudinal MRI ( 0.795 vs 0.774$)$ was modest, although both techniques represented an increase as compared to other published predictions of $\mathrm{MCI}$ conversion to $\mathrm{AD}$. If the longitudinal MRI is otherwise available, it seems evident that the incremental improvement in predictive accuracy would justify its use. It is unclear, however, that without other reasons for performing a 1-year follow up MRI, this increase in predictive accuracy would represent a new indication from a cost-effectiveness perspective. Thus, a comprehensive cost-benefit model analysis would be useful in this area.

The study used only anatomical MRI data. Multiparametric MRI (such as diffusion-tensor imaging, task functional MRI and resting-state MRI) will be incorporated into these models in the future. Similarly, other modalities such at Positron Emission Tomography (PET) and nonimaging clinical data can also be included in the model. Further studies will need to apply this approach to other datasets to improve generalizability. Future studies should investigate MCI to $\mathrm{AD}$ conversion at 1,2 and 5 years post-diagnosis.

Our model is a predictive model approach that employs machine learning based on whole-brain anatomical MRI to predict MCI to AD conversion. Future studies will need to compare different predictive models including those that predict $\mathrm{MCI}$ to $\mathrm{AD}$ conversion based on extracted volume and cortical thickness as obtained using tools such as FastSurfer (Henschel et al. 2020). To do so, we will first systematically explore various methods to extract volume and cortical thickness, explore various approaches (such neural networks and support vector machines) to predict MCI to ADC conversion, and use these methods to do head-to-head comparisons on the same datasets. 
452 Deep survival analysis (Ranganath et al. 2016) has been applied to the prediction of conversion 453 to AD. Nakagawa et al. used deep survival analysis to model the prediction of conversion from 454 either MCI or NC subjects to AD using volumetric data from MRI (Nakagawa et al. 2020). A 455 future extension of this analysis should investigate the use of data from the CNN models, both

\section{Conclusions}

461

462

463

464

465

466

467

468

469

470

471

472

473

474

475

476

477

478

479

480

481

482

483

484

485

486

487

488

489

490

491

492

493

This is the first convolutional neural network model using longitudinal and whole-brain 3D MRIs without extracting regional brain volumes or cortical thicknesses to predict future MCI to $\mathrm{AD}$ conversion. This framework set the stage for further studies of additional data time points, different image types, and non-image data to further improve prediction accuracy of MCI to AD conversion. Accurate prognosis could lead to better management of the diseases, thereby improving the quality of life.

\section{References}

Alaa AM, Bolton T, Di Angelantonio E, Rudd JHF, and van der Schaar M. 2019. Cardiovascular disease risk prediction using automated machine learning: A prospective study of 423,604 UK Biobank participants. PLoS One 14:e0213653. 10.1371/journal.pone.0213653

Basaia S, Agosta F, Wagner L, Canu E, Magnani G, Santangelo R, Filippi M, and Alzheimer's Disease Neuroimaging I. 2019. Automated classification of Alzheimer's disease and mild cognitive impairment using a single MRI and deep neural networks. Neuroimage Clin 21:101645. 10.1016/j.nicl.2018.101645

Bhagwat N, Viviano JD, Voineskos AN, Chakravarty MM, and Alzheimer's Disease Neuroimaging I. 2018. Modeling and prediction of clinical symptom trajectories in Alzheimer's disease using longitudinal data. PLoS Comput Biol 14:e1006376. 10.1371/journal.pcbi.1006376

Brun A, and Gustafson L. 1976. Distribution of cerebral degeneration in Alzheimer's disease. A clinico-pathological study. Arch Psychiatr Nervenkr (1970) 223:15-33. 10.1007/BF00367450

Cheng D, Liu M, Fu J, and Wang Y. 2017. Classification of MR brain images by combination of multi-CNNs for AD diagnosis. Ninth International Conference on Digital Image Processing (ICDIP 2017): SPIE.

de Bruijne M. 2016. Machine learning approaches in medical image analysis: From detection to diagnosis. Med Image Anal 33:94-97. 10.1016/j.media.2016.06.032

Epperly T, Dunay MA, and Boice JL. 2017. Alzheimer Disease: Pharmacologic and Nonpharmacologic Therapies for Cognitive and Functional Symptoms. Am Fam Physician 95:771-778. 
494

495

496

497

498

499

500

501

502

503

504

505

506

507

508

509

510

511

512

513

514

515

516

517

518

519

520

521

522

523

524

525

526

527

528

529

530

531

532

533

534

535

536

537

538

Erickson BJ, Korfiatis P, Akkus Z, and Kline TL. 2017. Machine Learning for Medical Imaging. Radiographics 37:505-515. 10.1148/rg.2017160130

Graupe D. 2013. Principles of artificial neural networks. New Jersey: World Scientific.

Guo X, Wang Z, Li K, Li Z, Qi Z, Jin Z, Yao L, and Chen K. 2010. Voxel-based assessment of gray and white matter volumes in Alzheimer's disease. Neurosci Lett 468:146-150. 10.1016/j.neulet.2009.10.086

Harris M, Qi A, Jeagal L, Torabi N, Menzies D, Korobitsyn A, Pai M, Nathavitharana RR, and Ahmad Khan F. 2019. A systematic review of the diagnostic accuracy of artificial intelligence-based computer programs to analyze chest x-rays for pulmonary tuberculosis. PLoS One 14:e0221339. 10.1371/journal.pone.0221339

He K, Zhang X, Ren S, and Sun J. 2016. Deep residual learning for image recognition. Proceedings of the IEEE conference on computer vision and pattern recognition. $\mathrm{p}$ 770778.

Henschel L, Conjeti S, Estrada S, Diers K, Fischl B, and Reuter M. 2020. Fastsurfer-a fast and accurate deep learning based neuroimaging pipeline. Neuroimage 219:117012.

Hirono N, Mori E, Ishii K, Ikejiri Y, Imamura T, Shimomura T, Hashimoto M, Yamashita H, and Sasaki M. 1998. Hypofunction in the posterior cingulate gyrus correlates with disorientation for time and place in Alzheimer's disease. J Neurol Neurosurg Psychiatry 64:552-554. 10.1136/jnnp.64.4.552

Itzcovich I. 2018. DeepBrain Extractor. GitHub.

Jak AJ, Bondi MW, Delano-Wood L, Wierenga C, Corey-Bloom J, Salmon DP, and Delis DC. 2009. Quantification of five neuropsychological approaches to defining mild cognitive impairment. Am J Geriatr Psychiatry 17:368-375. 10.1097/JGP.0b013e31819431d5

Jenkinson M, Beckmann CF, Behrens TE, Woolrich MW, and Smith SM. 2012. Fsl. Neuroimage 62:782-790. 10.1016/j.neuroimage.2011.09.015

Johnson KA, Fox NC, Sperling RA, and Klunk WE. 2012. Brain imaging in Alzheimer disease. Cold Spring Harb Perspect Med 2:a006213. 10.1101/cshperspect.a006213

Johnson KW, Torres Soto J, Glicksberg BS, Shameer K, Miotto R, Ali M, Ashley E, and Dudley JT. 2018. Artificial Intelligence in Cardiology. J Am Coll Cardiol 71:2668-2679. 10.1016/j.jacc.2018.03.521

Jones BF, Barnes J, Uylings HB, Fox NC, Frost C, Witter MP, and Scheltens P. 2006. Differential regional atrophy of the cingulate gyrus in Alzheimer disease: a volumetric MRI study. Cereb Cortex 16:1701-1708. 10.1093/cercor/bhj105

Kao YH, Chou MC, Chen CH, and Yang YH. 2019. White Matter Changes in Patients with Alzheimer's Disease and Associated Factors. J Clin Med 8. 10.3390/jcm8020167

Kingma DP, and Ba JA. 2019. A method for stochastic optimization. arXiv 2014. arXiv preprint arXiv:14126980 434.

Korolev S, Safiullin A, Belyaev M, and Dodonova Y. 2017. Residual and plain convolutional neural networks for 3D brain MRI classification. 2017 IEEE 14th International Symposium on Biomedical Imaging (ISBI 2017): IEEE. p 835-838.

Krizhevsky A, Sutskever I, and Hinton GE. 2012. Imagenet classification with deep convolutional neural networks. Advances in neural information processing systems. $p$ 1097-1105.

Lecun Y, Bottou L, Bengio Y, and Haffner P. 1998. Gradient-based learning applied to document recognition. Proceedings of the IEEE 86:2278-2324. 10.1109/5.726791

Peer] Comput. Sci. reviewing PDF | (CS-2020:10:54832:2:0:NEW 26 Apr 2021) 
539

540

541

542

543

544

545

546

547

548

549

550

551

552

553

554

555

556

557

558

559

560

561

562

563

564

565

566

567

568

569

570

571

572

573

574

575

576

577

578

579

580

581

582
Li MD, Chang K, Bearce B, Chang CY, Huang AJ, Campbell JP, Brown JM, Singh P, Hoebel KV, and Erdoğmuş D. 2020. Siamese neural networks for continuous disease severity evaluation and change detection in medical imaging. NPJ digital medicine 3:1-9.

Lian C, Liu M, Zhang J, and Shen D. 2018. Hierarchical Fully Convolutional Network for Joint Atrophy Localization and Alzheimer's Disease Diagnosis Using Structural MRI. IEEE Trans Pattern Anal Mach Intell 42:880-893. 10.1109/TPAMI.2018.2889096

Lin W, Tong T, Gao Q, Guo D, Du X, Yang Y, Guo G, Xiao M, Du M, Qu X, and TAsDNI. 2018. Convolutional Neural Networks-Based MRI Image Analysis for the Alzheimer's Disease Prediction From Mild Cognitive Impairment. Frontiers in Neuroscience 12. 10.3389/fnins.2018.00777

Lip GY, Nieuwlaat R, Pisters R, Lane DA, and Crijns HJ. 2010. Refining clinical risk stratification for predicting stroke and thromboembolism in atrial fibrillation using a novel risk factor-based approach: the euro heart survey on atrial fibrillation. Chest 137:263-272. 10.1378/chest.09-1584

Liu M, Zhang J, Adeli E, and Shen D. 2018. Landmark-based deep multi-instance learning for brain disease diagnosis. Med Image Anal 43:157-168. 10.1016/j.media.2017.10.005

McKhann GM, Knopman DS, Chertkow H, Hyman BT, Jack CR, Jr., Kawas CH, Klunk WE, Koroshetz WJ, Manly JJ, Mayeux R, Mohs RC, Morris JC, Rossor MN, Scheltens P, Carrillo MC, Thies B, Weintraub S, and Phelps CH. 2011. The diagnosis of dementia due to Alzheimer's disease: recommendations from the National Institute on AgingAlzheimer's Association workgroups on diagnostic guidelines for Alzheimer's disease. Alzheimers Dement 7:263-269. 10.1016/j.jalz.2011.03.005

Nakagawa T, Ishida M, Naito J, Nagai A, Yamaguchi S, Onoda K, and Initiative AsDN. 2020. Prediction of conversion to Alzheimer's disease using deep survival analysis of MRI images. Brain communications 2:fcaa057.

O'Mahony C, Jichi F, Pavlou M, Monserrat L, Anastasakis A, Rapezzi C, Biagini E, Gimeno JR, Limongelli G, McKenna WJ, Omar RZ, Elliott PM, and Hypertrophic Cardiomyopathy Outcomes I. 2014. A novel clinical risk prediction model for sudden cardiac death in hypertrophic cardiomyopathy (HCM risk-SCD). Eur Heart J 35:2010-2020. 10.1093/eurheartj/eht439

Ostertag C, Beurton-Aimar M, and Urruty T. 2019. 3D-SiameseNet to analyze brain MRI. 10th International Conference on Pattern Recognition Systems (ICPRS-2019): IET. p 18-23.

Pan SJ, and Yang Q. 2010. A Survey on Transfer Learning. IEEE Transactions on Knowledge and Data Engineering 22:1345-1359. 10.1109/TKDE.2009.191

Petersen RC, Smith GE, Waring SC, Ivnik RJ, Tangalos EG, and Kokmen E. 1999. Mild cognitive impairment: clinical characterization and outcome. Arch Neurol 56:303-308. 10.1001/archneur.56.3.303

Pini L, Pievani M, Bocchetta M, Altomare D, Bosco P, Cavedo E, Galluzzi S, Marizzoni M, and Frisoni GB. 2016. Brain atrophy in Alzheimer's Disease and aging. Ageing Res Rev 30:25-48. 10.1016/j.arr.2016.01.002

Ranganath R, Perotte A, Elhadad N, and Blei D. 2016. Deep Survival Analysis. In: Finale D-V, Jim F, David K, Byron W, and Jenna W, editors. Proceedings of the 1st Machine Learning for Healthcare Conference. Proceedings of Machine Learning Research: PMLR. p 101--114.

Peer] Comput. Sci. reviewing PDF | (CS-2020:10:54832:2:0:NEW 26 Apr 2021) 
583

584

585

586

587

588

589

590

591

592

593

594

595

596

597

598

599

600

601

602

603

604

605

606

607

608

609

610

611

612

613

614
Selvaraju RR, Cogswell M, Das A, Vedantam R, Parikh D, and Batra D. 2017. Grad-cam: Visual explanations from deep networks via gradient-based localization. Proceedings of the IEEE international conference on computer vision. $\mathrm{p}$ 618-626.

Shmulev Y, and Belyaev M. 2018. Predicting Conversion of Mild Cognitive Impairments to Alzheimer's Disease and Exploring Impact of Neuroimaging. In: Stoyanov D, Taylor Z, Ferrante E, Dalca AV, Martel A, Maier-Hein L, Parisot S, Sotiras A, Papiez B, Sabuncu MR, and Shen L, editors. Graphs in Biomedical Image Analysis and Integrating Medical Imaging and Non-Imaging Modalities. Cham: Springer International Publishing. p 83-91. Simonyan K, and Zisserman A. 2014. Very deep convolutional networks for large-scale image recognition. arXiv preprint arXiv: 14091556.

Sled JG, Zijdenbos AP, and Evans AC. 1998. A nonparametric method for automatic correction of intensity nonuniformity in MRI data. IEEE transactions on medical imaging 17:87-97.

Smith CD, Snowdon DA, Wang H, and Markesbery WR. 2000. White matter volumes and periventricular white matter hyperintensities in aging and dementia. Neurology 54:838842. 10.1212/wnl.54.4.838

Virtanen P, Gommers R, Oliphant TE, Haberland M, Reddy T, Cournapeau D, Burovski E, Peterson P, Weckesser W, Bright J, van der Walt SJ, Brett M, Wilson J, Millman KJ, Mayorov N, Nelson ARJ, Jones E, Kern R, Larson E, Carey CJ, Polat I, Feng Y, Moore EW, VanderPlas J, Laxalde D, Perktold J, Cimrman R, Henriksen I, Quintero EA, Harris CR, Archibald AM, Ribeiro AH, Pedregosa F, van Mulbregt P, and SciPy C. 2020. SciPy 1.0: fundamental algorithms for scientific computing in Python. Nat Methods 17:261272. 10.1038/s41592-019-0686-2

Wen J, Thibeau-Sutre E, Diaz-Melo M, Samper-Gonzalez J, Routier A, Bottani S, Dormont D, Durrleman S, Burgos N, Colliot O, Alzheimer's Disease Neuroimaging I, Australian Imaging B, and Lifestyle flagship study of a. 2020. Convolutional neural networks for classification of Alzheimer's disease: Overview and reproducible evaluation. Med Image Anal 63:101694. 10.1016/j.media.2020.101694

Zagoruyko S, and Komodakis N. 2016. Wide residual networks. arXiv preprint arXiv: 160507146.

Zhang Y, and Wang S. 2015. Detection of Alzheimer's disease by displacement field and machine learning. PeerJ 3:e1251. 10.7717/peerj.1251 


\section{Figure 1}

Overview of experimental design.

$A D$ and NC MRI data were first trained to obtain weights (a classification task) for transfer learning (blue). After training, the weights are transferred to the prediction task (green) to predict whether patients will remain stable or progress within three years. Two different transfer learning methods were studied. With zero-shot, no further training was performed after the transfer, so the $\mathrm{MCl}$ images were analyzed for prediction by the network with the same weights copied over from the classification task. With fine-tuning, after weights are copied over from the classification task for initialization, additional training is performed against the $\mathrm{MCl}$ image data.

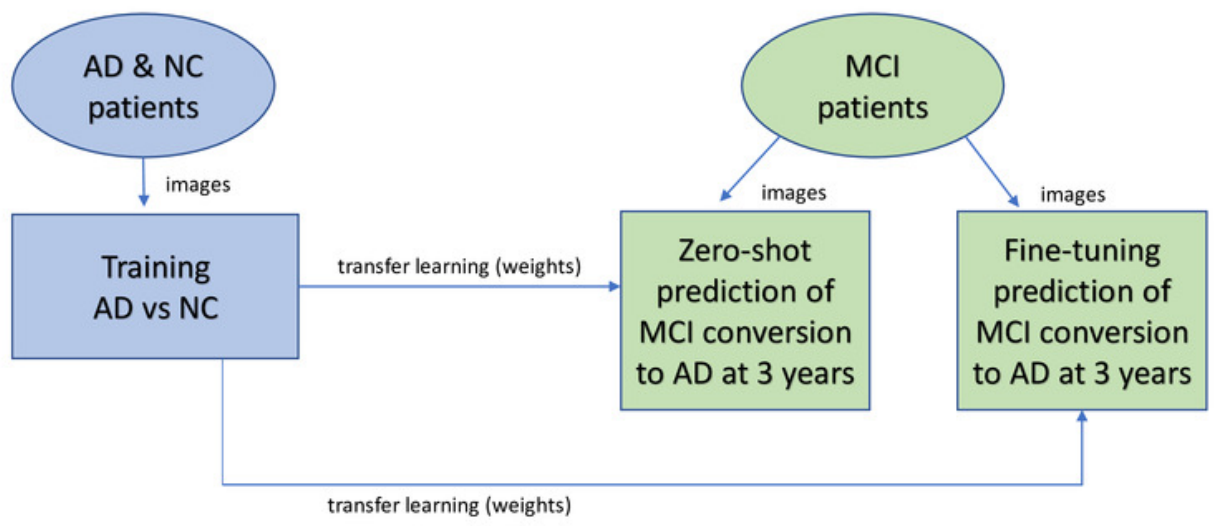




\section{Figure 2}

Single and dual time point CNN architecture.

(A) Single timepoint CNN. For classification, input consisted of a single timepoint full-subject 3D MRI of patients diagnosed at baseline as either $A D$ or $C N$, and output was binary classification of $A D$ vs $C N$. For prediction, input was a single timepoint full-subject 3D MRI of patients diagnosed as $\mathrm{MCl}$ and output was a binary prediction of whether the patient progressed $(\mathrm{pMCl})$ or remained stable $(\mathrm{sMCl}) 3$ years later. (B) Dual timepoint CNN. Input included 3D MRI images obtained at both baseline and 12 months, with the patient population and output categories identical than those used for single timepoint for classification and prediction. Both kinds of networks began with a series of convolutional blocks, followed by flattening into one or more fully connected layers ending in a final binary choice of classification or prediction.

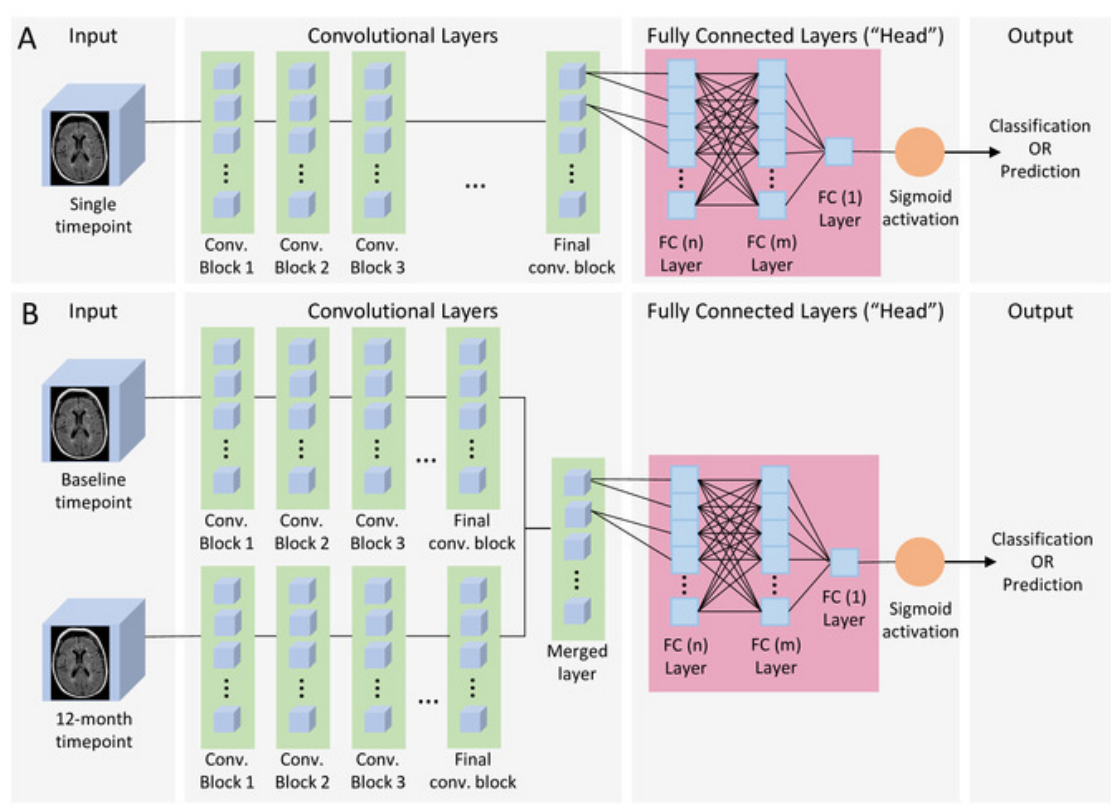




\section{Figure 3}

Sequential, residual with bottleneck, and wide residual CNN blocks.

The convolutional layers portion of the network was organized as a series of blocks, each one with an increasing number $\mathrm{K}$ of activation maps (width), and with a corresponding decrease in resolution obtained by either pooling or stride during convolution. The figures detail the individual layers that compose a single block. (A) Sequential convolutional block. Each block was composed of a single $3 \times 3 \times 3$ convolution, followed by batch normalization, ReLU activation, and max pooling to reduce the resolution. (B)Residual bottleneck with preactivation convolutional block. Convolutions were preceded by batch normalization and ReLU activation. Two bottleneck $3 \times 3 \times 3$ convolutions have a width of $\mathrm{K} / 4$ followed by a final $1 \times 1 \times 1$ convolution with $\mathrm{K}$ width. In parallel the skip residual used a $1 \times 1 \times 1$ convolution to match the width and resolution. In this architecture the first residual block was preceded by an initial batch normalization followed by a single $5 \times 5 \times 5$ convolution, plus one final batch normalization and ReLU activation after the last block (not shown). (C) Wide Residual Network convolutional block. In this architecture the batch normalization and activations occured after the convolutional layers. Each block had two $3 \times 3 \times 3$ convolutional layers with 3D spatial dropout in between, plus a $1 \times 1 \times 1$ skip residual convolution to match width and resolution. 

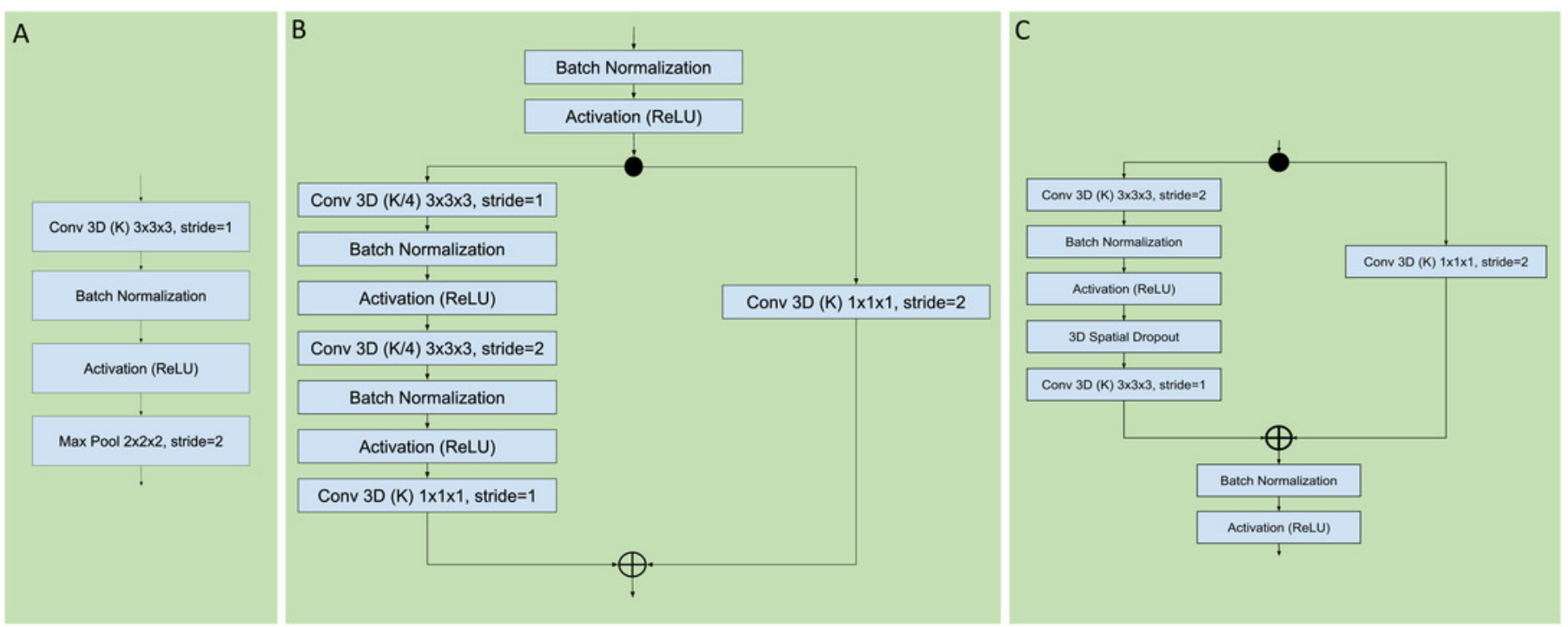


\section{Figure 4}

Three head architectures.

(A) 3D global maximum pooling fully connected block. The global pooling inherently flattened the nodes into a fully connected layer with $\mathrm{N}$ nodes directly followed by the final binary classifier layer. (B) Long fully connected block. After flattening into a layer of $\mathrm{N}$ nodes, there are two sets of fully connected (size 2048 and 1024), batch normalization, and leaky ReLU activation layers separated by a single dropout layer, before the final binary classifier. (C) Medium fully connected block. Initial 3D max pooling is followed by flattening into a fully connected layer of size $\mathrm{N}$ followed by an additional fully connected layer of size 128 and ReLU activation.
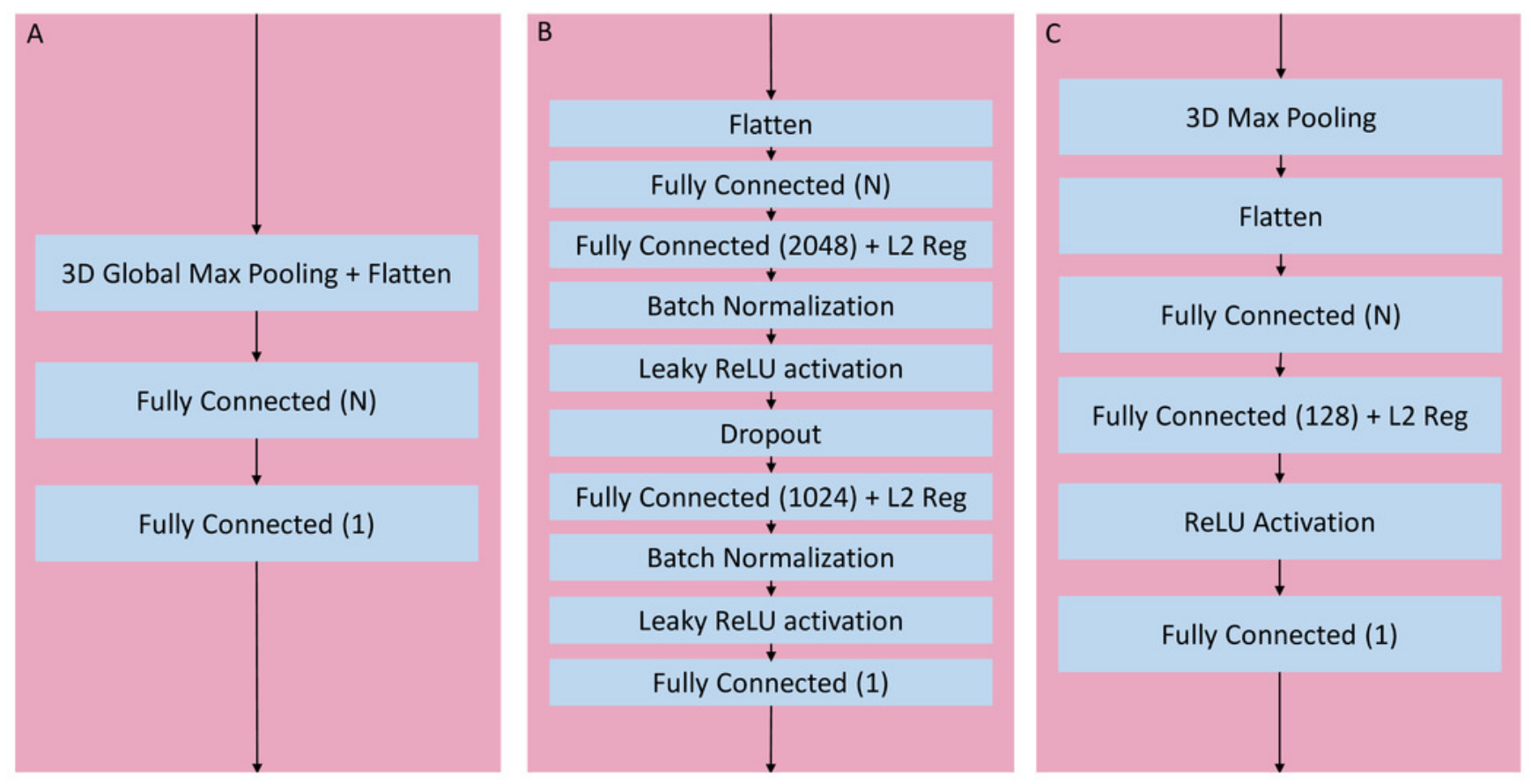


\section{Figure 5}

Training curves during classification.

Loss and Accuracy curves during training for both training and validation sets. For sequential network and single timepoint, (A) loss, (B) accuracy. For wide residual network and dual timepoints, (C) loss, (D) accuracy. Solid lines are smoothed with 0.8 factor and faint lines show the unsmoothed values for each epoch.
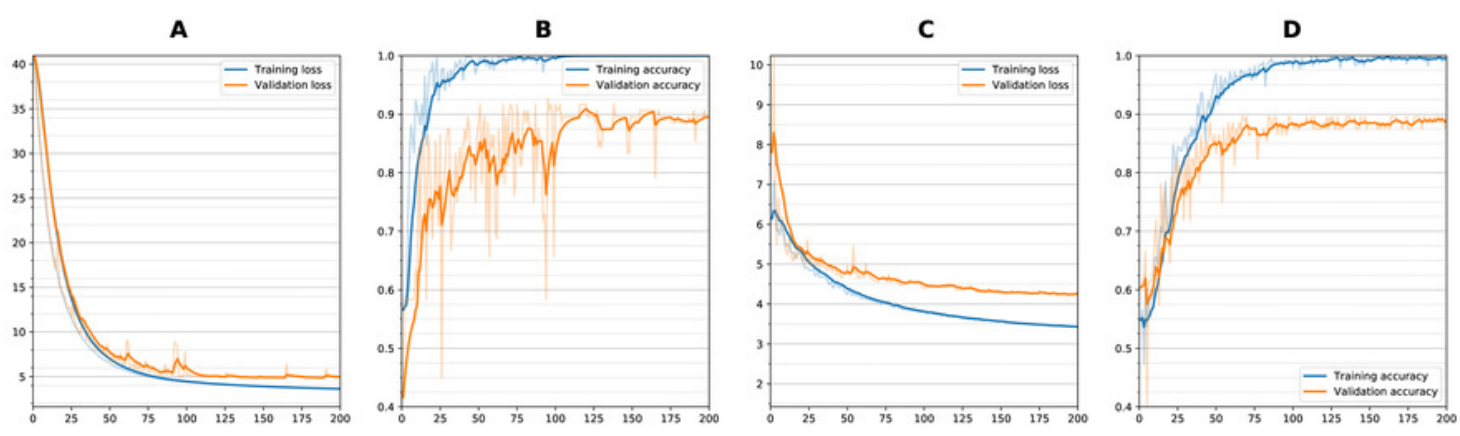
Figure 6

Training curve during fine tuning for prediction.

(A) loss function per epoch and (B) accuracy per epoch) during transfer learning fine tuning (sequential dual channel). Weights were initialized after training with AD vs. NC and then frozen at the convolutional layers, then additional training performed with the $s \mathrm{MCl} v \mathrm{vs} . \mathrm{pMCl}$ data. There is an initial reduction in loss which stabilizes after 10 epochs, with no increase in accuracy.

A

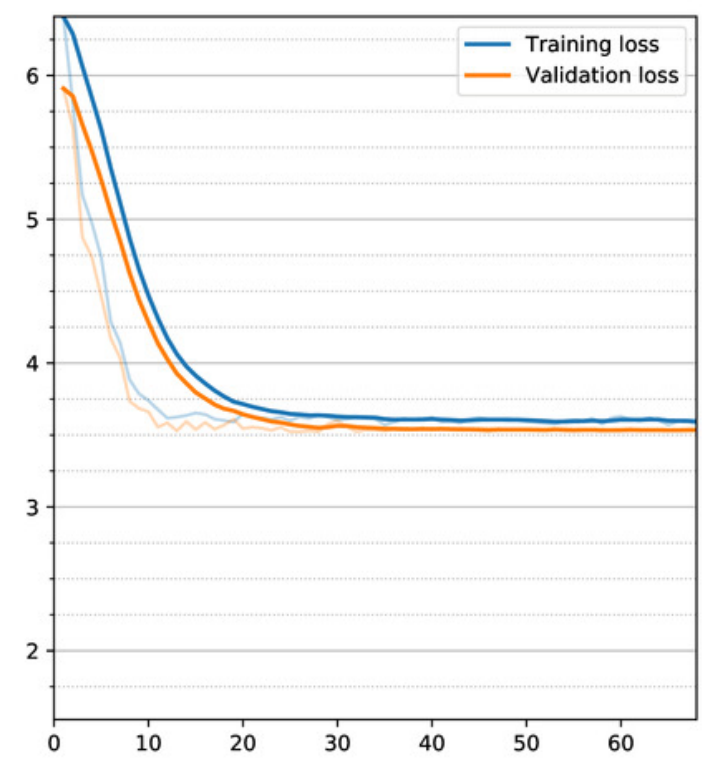

B

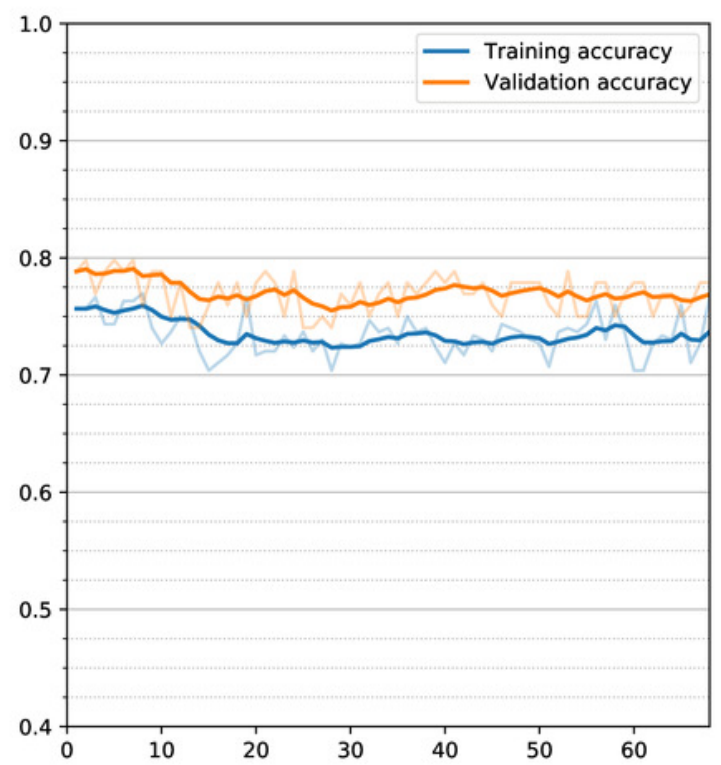




\section{Figure 7}

Heatmap visualization for 10 patients.

3D Grad-CAM heatmaps from the wide residual dual channel network used to predict conversion of $\mathrm{MCl}$ to $\mathrm{AD}$. Heat maps were superimposed on individual patient's anatomical MRI of 10 patients.A areas in bright yellow-orange (low to high) color corresponding to voxels with the gradient based on 3D Grad-CAM algorithm at the convolutional layer around 20 pixel resolution. 


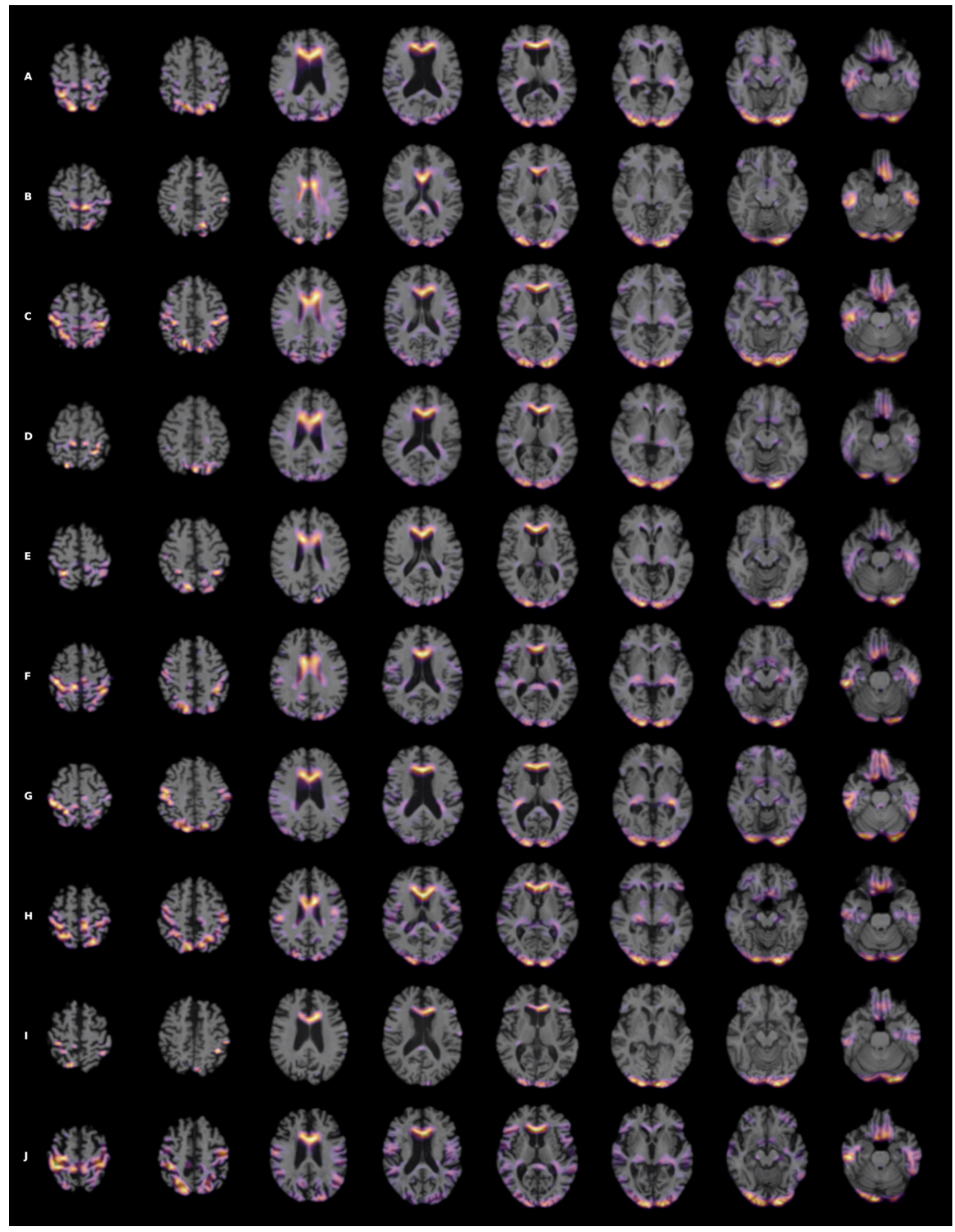




\section{Table 1 (on next page)}

Summary of participant demographics 
1

\begin{tabular}{|l|c|c|c|}
\hline & $\mathrm{N}$ & Age (years) & Gender \\
\hline $\mathrm{NC}$ & 320 & $75.7 \pm 5.6[60.9,90.8]$ & $160 \mathrm{~F} / 160 \mathrm{M}$ \\
\hline $\mathrm{AD}$ & 237 & $75.9 \pm 7.9[56.1,92.0]$ & $108 \mathrm{~F} / 129 \mathrm{M}$ \\
\hline $\mathrm{sMCl}$ & 333 & $72.7 \pm 7.6[56.1,89.8]$ & $137 \mathrm{~F} / 196 \mathrm{M}$ \\
\hline $\mathrm{pMCl}$ & 221 & $75.2 \pm 7.2[55.6,89.5]$ & $92 \mathrm{~F} / 129 \mathrm{M}$ \\
\hline
\end{tabular}

$2 \mathrm{NC}=$ normal cognition

$3 \mathrm{AD}=$ Alzheimer's disease

$4 \mathrm{sMCl}=$ stable mild cognitive impairment

$5 \mathrm{pMCl}=$ progressive mild cognitive impairment

$6 \quad \mathrm{~F}=$ Female

$7 \mathrm{M}=$ Male

8 Age values are mean \pm standard deviation [range] 


\section{Table 2 (on next page)}

AD vs NC classification to generate weights.

Balanced accuracy and area under the receiver operating characteristic curve of the validation and test datasets obtained using single and dual time point networks with sequential, bottleneck residual and wide residual CNN blocks. Dual timepoint networks were twin (equal structure), non-Siamese (separate weights) and merged using concatenation. 
1

\begin{tabular}{|c|l|c|c|c|c|}
\hline \multirow{2}{*}{$\begin{array}{l}\text { Model } \\
\text { Convolution } \\
\text { Style }\end{array}$} & \multicolumn{2}{|c|}{ Validation mean \pm SD } & \multicolumn{2}{c|}{ Test mean \pm SD } \\
\cline { 2 - 6 } & BA & AUC & BA & AUC \\
\hline \multirow{5}{*}{ Single } & Sequential & $0.854 \pm 0.027$ & $0.918 \pm 0.018$ & $\mathbf{0 . 8 6 0 \pm 0 . 0 1 6}$ & $0.922 \pm 0.005$ \\
\cline { 2 - 6 } & $\begin{array}{l}\text { Bottleneck } \\
\text { Residual }\end{array}$ & $0.689 \pm 0.020$ & $0.774 \pm 0.017$ & $0.727 \pm 0.051$ & $0.782 \pm 0.052$ \\
\cline { 2 - 6 } & Wide Residual & $0.835 \pm 0.025$ & $0.903 \pm 0.027$ & $0.840 \pm 0.017$ & $0.917 \pm 0.006$ \\
\hline \multirow{3}{*}{ Dual } & Sequential & $0.855 \pm 0.014$ & $0.938 \pm 0.007$ & $0.876 \pm 0.010$ & $0.937 \pm 0.012$ \\
\cline { 2 - 6 } & $\begin{array}{l}\text { Bottleneck } \\
\text { Residual }\end{array}$ & $0.772 \pm 0.046$ & $0.865 \pm 0.037$ & $0.800 \pm 0.045$ & $0.869 \pm 0.043$ \\
\cline { 2 - 6 } & Wide Residual & $0.856 \pm 0.025$ & $0.942 \pm 0.012$ & $\mathbf{0 . 8 8 7} \pm \mathbf{0 . 0 0 9}$ & $0.933 \pm 0.003$ \\
\hline
\end{tabular}

$2 \quad \mathrm{BA}=$ balanced accuracy.

$3 \quad \mathrm{AUC}=$ area under the receiver operating characteristic curve

4 Best test average BAs are highlighted in bold for single channel (sequential) and dual channel 5 (wide residual) 


\section{Table 3(on next page)}

Prediction of $A D$ at 3 years.

Results (BA and AUC mean and standard deviation) of prediction using zero-shot and finetuning. For single-timepoint networks, resluts are shown using both the baseline and the 1year MRIs. For zero-shot learning, each of the 4-fold classification trained weights were used as-is against each of the 4 validation fold sets for prediction (16 attempts) and against the prediction test set ( 4 attempts, with best result also shown). For fine-tuning, the weights from the best test zero-shot result were used as starting weights for training against each of the 4fold validation sets. 


\begin{tabular}{|c|c|c|c|c|c|c|c|c|c|}
\hline & \multirow{3}{*}{$\begin{array}{c}\text { Model } \\
\text { Convolution } \\
\text { style }\end{array}$} & \multicolumn{4}{|c|}{ Validation mean $\pm S D$} & \multicolumn{4}{|c|}{ Test mean $\pm S D$} \\
\hline & & \multicolumn{2}{|c|}{ Zero-shot } & \multicolumn{2}{|c|}{ Fine-tuning } & \multicolumn{2}{|c|}{ Zero-shot } & \multicolumn{2}{|c|}{ Fine-tuning } \\
\hline & & BA & AUC & BA & AUC & $\begin{array}{c}\text { BA } \\
\text { (best) }\end{array}$ & AUC & BA & AUC \\
\hline \multirow{2}{*}{$\begin{array}{l}\text { Single } \\
\text { (baseline) }\end{array}$} & Sequential & $\begin{array}{l}0.728 \quad \pm \\
0.043\end{array}$ & $\begin{array}{l}0.790 \pm \\
0.043\end{array}$ & $\begin{array}{l}0.746 \pm \\
0.0033\end{array}$ & $\begin{array}{l}0.805 \pm \\
0.028\end{array}$ & $\begin{array}{l}0.765 \\
0.021 \\
(0.79)\end{array}$ & $\begin{array}{l}0.831 \pm \\
0.015\end{array}$ & $\begin{array}{l}0.754 \\
0.026\end{array}$ & $\begin{array}{l}0.834 \pm \\
0.015\end{array}$ \\
\hline & $\begin{array}{l}\text { Wide } \\
\text { Residual }\end{array}$ & $\begin{array}{l}0.699 \quad \pm \\
0.034\end{array}$ & $\begin{array}{l}0.775 \quad \pm \\
0.034\end{array}$ & $\begin{array}{l}0.700 \pm \\
0.017\end{array}$ & $\begin{array}{l}0.774 \pm \\
0.022\end{array}$ & $\begin{array}{l}0.706 \\
0.031 \\
(0.79)\end{array}$ & $\begin{array}{l}0.816 \pm \\
0.024\end{array}$ & $\begin{array}{ll}0.717 & \pm \\
0.030\end{array}$ & $\begin{array}{l}0.816 \pm \\
0.024\end{array}$ \\
\hline \multirow{2}{*}{$\begin{array}{l}\text { Single } \\
\text { (1 year) }\end{array}$} & Sequential & $\begin{array}{l}0.750 \quad \pm \\
0.038\end{array}$ & $\begin{array}{l}0.807 \quad \pm \\
0.039\end{array}$ & $\begin{array}{l}0.733 \pm \\
0.050\end{array}$ & $\begin{array}{l}0.814 \pm \\
0.042\end{array}$ & $\begin{array}{l}0.774 \\
0.013 \\
(0.79)\end{array}$ & $\begin{array}{l}0.857 \pm \\
0.012\end{array}$ & $\begin{array}{ll}0.728 & \pm \\
0.008\end{array}$ & $\begin{array}{l}0.836 \pm \\
0.001\end{array}$ \\
\hline & \begin{tabular}{|l} 
Wide \\
Residual
\end{tabular} & $\begin{array}{l}0.729 \\
0.038\end{array}$ & $\begin{array}{l}0.799 \\
0.028\end{array}$ & $\begin{array}{l}0.704 \pm \\
0.037\end{array}$ & $\begin{array}{l}0.782 \pm \\
0.033\end{array}$ & $\begin{array}{l}0.743 \\
0.029 \\
(0.77)\end{array}$ & $\begin{array}{l}0.834 \pm \\
0.020\end{array}$ & $\begin{array}{ll}0.719 & \pm \\
0.007\end{array}$ & $\begin{array}{l}0.803 \pm \\
0.001\end{array}$ \\
\hline \multirow{2}{*}{ Dual } & Sequential & $\begin{array}{l}0.751 \quad \pm \\
0.027\end{array}$ & $\begin{array}{l}0.808 \\
0.029\end{array}$ & $\begin{array}{l}0.712 \pm \\
0.027\end{array}$ & $\begin{array}{l}0.772 \pm \\
0.039\end{array}$ & $\begin{array}{l}0.795 \\
0.010 \\
(0.80)\end{array}$ & $\begin{array}{l}0.874 \pm \\
0.009\end{array}$ & $\begin{array}{l}0.739 \\
0.012\end{array}$ & $0.828 \pm$ \\
\hline & $\begin{array}{l}\text { Wide } \\
\text { Residual }\end{array}$ & $\begin{array}{l}0.727 \\
0.038\end{array}$ & $\begin{array}{l}0.806 \pm \\
0.033\end{array}$ & $\begin{array}{l}0.719 \pm \\
0.018\end{array}$ & $\begin{array}{l}0.801 \pm \\
0.032\end{array}$ & $\begin{array}{l}0.753 \\
0.034 \\
(0.79)\end{array}$ & $\begin{array}{l}0.842 \pm \\
0.010\end{array}$ & $\begin{array}{l}0.775 \\
0.003\end{array}$ & $\begin{array}{l}0.834 \pm \\
0.001\end{array}$ \\
\hline
\end{tabular}

1

$2 \quad A \cup C=$ area under the receiver operating characteristic curve

3 BA = balanced accuracy

$4 \quad \mathrm{SD}=$ standard deviation 